Article

\title{
Comparative Investigations of Tidal Current Velocity Prediction Considering Effect of Multi-Layer Current Velocity
}

\author{
Bo Feng, Peng Qian, Yulin Si, Xiaodong Liu, Haixiao Yang, Huisheng Wen and Dahai Zhang *(D) \\ Ocean College, Zhejiang University, Hangzhou 310000, China; bofeng@zju.edu.cn (B.F.); \\ qianpeng18@sina.com (P.Q.); yulinsi@zju.edu.cn (Y.S.); liuxiaodong@zju.edu.cn (X.L.); \\ 21934153@zju.edu.cn (H.Y.); 21934134@zju.edu.cn (H.W.) \\ * Correspondence: zhangdahai@zju.edu.cn
}

Received: 31 October 2020; Accepted: 3 December 2020; Published: 4 December 2020

\begin{abstract}
Accurate tidal current prediction plays a critical role with increasing utilization of tidal energy. The classical prediction approach of the tidal current velocity adopts the harmonic analysis (HA) method. The performance of the HA approach is not ideal to predict the high frequency components of tidal currents due to the lack of capability processing the non-astronomic factor. Recently, machine learning algorithms have been applied to process the non-astronomic factor in the prediction of tidal current. In this paper, a tidal current velocity prediction considering the effect of the multi-layer current velocity method is proposed. The proposed method adopts three machine learning algorithms to establish the prediction models for comparative investigations, namely long-short term memory (LSTM), back-propagation (BP) neural network, and the Elman regression network. In the case study, the tidal current data collected from the real ocean environment were used to validate the proposed method. The results show that the proposed method combined with the LSTM algorithm had higher accuracy than both the commercial tidal prediction tool (UTide) and the other two algorithms. This paper presents a novel tidal current velocity prediction considering the effect of the multi-layer current velocity method, which improves the accuracy of the power flow prediction and contributes to the research in the field of tidal current velocity prediction and the capture of tidal energy.
\end{abstract}

Keywords: tidal current prediction; multilayer current velocity; UTide; machine learning; turbulence flow

\section{Introduction}

Tidal currents take place simultaneously with the rise and fall of the tide. The vertical motion of tides leads the water to move horizontally, which generates tidal currents. Tidal current energy is the marine energy from the seawater, occurring in submarine channels and straits [1-3]. Compared with other renewable energy, tidal current energy has the advantages of predictability, stability, and high-density [4-6]. In contrast to the conventional generations, tidal current energy still suffers a problem of uncertainty, which interferes with the reliability of the power grid [7-9]. As the grid-connected power generation system has a very high demand for power quality, and the power fluctuation of tidal energy would significantly increase the difficulty of power management between power generation and load side [10]. With the development and utilization of tidal current energy, it would be desirable to develop an accurate short-term tidal current prediction method.

There are two factors that affect tidal currents including the astronomic factor and the random factor. The astronomic factor is determined by gravitational force. The astronomic factor of tidal currents is a kind of periodic component because of the periodic motion of the Moon and the Sun; 
the random factor is affected by variations of the turbulence flow, the wind, and the temperature, etc. Hence, the random factor of tidal currents generates random components. In terms of the tidal current prediction method, there are two general types of methods, namely the harmonic analysis method and machine learning based approach.

The harmonic analysis (HA) method, which was proposed by Professor G. H. Darwin [11], is the most widely used method in tidal current prediction. In the HA method, tidal currents are regarded as a combination of various periodic components. The HA method has therefore been applied in stationary tidal current analysis; the information of tidal dynamics can be calculated from the HA method [12]. However, the HA method has some disadvantages that limit its application. The accuracy of the HA method output is dependent on the component selection [13]. In addition to the component selection issue, the noise associated with the problem is another issue that poses a challenge for the HA method. For example, the turbulence flow is very difficult to predict by using the HA method. From Jay's research results [14], it shows that the HA method is also not suitable to be applied in non-stationary data analysis.

Apart from the HA method, machine learning methods have been utilized by many researchers in the renewable energy system [15-18]. Artificial neural network (ANN) is a type of classical machine learning method [19-22]. Due to high robust performance in noisy environments, ANN-based methods are suitable to solve noise associated with issues [23]. However, the performances of traditional ANN-based methods are not ideal in some complicated applications [24]. With the development of artificial intelligence technology, the deep neural network (DNN) has shown great potential [25]. The long short-term memory (LSTM) algorithm is a branch of DNN algorithms, which enables the effective process of many types of time series signals [26,27]. Furthermore, the LSTM algorithm has an advantage of capturing long-term dependencies within a sequence [28]. Essentially, the LSTM algorithm is a kind of special recurrent neural network (RNN), which is more suitable to process long-term dependence problems [29]. This feature of the LSTM algorithm makes it suitable to be applied in tidal current prediction because the main component of tidal currents is a kind of sequential periodic wave.

\section{Study Area}

It is clear that the tidal current prediction model, considering both the random factor and astronomic factor, is able to increase the prediction accuracy. Hence, a novel tidal current velocity prediction considering the effect of multi-layer current velocity based on the machine learning method was proposed. By analysis of the effect of multi-layer current velocity, both the astronomic factor and random factor of the tidal current are considered in the prediction model. In previous research, little work considered the effect of multi-layer current velocity in tidal current prediction. Otherwise, all machine learning based tidal current prediction approaches have their own advantages and drawbacks. These have been developed for different renewable energy prediction systems for decades. Hence, it is very useful to carefully evaluate and compare these methods before their applications, which help to select the appropriate strategy for a given case.

The main contributions of this paper are described as follows:

- A tidal current velocity prediction considering the effect of the multi-layer current velocity prediction method is proposed, which enables the effect of turbulence flow to be reduced in prediction accuracy;

- The LSTM algorithm method is applied in tidal current prediction; and

- Comparative investigations of machine learning based approaches on tidal current prediction are given.

The remainder of this paper is organized as follows. Section 2 introduces the proposed tidal current velocity prediction considering the effect of the multi-layer current velocity method. Section 3 illustrates the traditional harmonic analysis method. Section 4 presents the machine learning based 
tidal current prediction algorithms. In Section 5, comparative investigations of machine learning based approaches for tidal current prediction are given by using the tidal current data. Conclusions and future work are at the end of this paper.

\section{Methodology}

\subsection{Tidal Current Velocity Prediction Considering Effect of Multi-Layer Current Velocity}

In previous research, little work had considered the effect of multi-layer current velocity in tidal current prediction. The tidal current velocity prediction generally pays attention to the tidal current velocity in a specific depth [30]. Figure 1 presents a tidal current velocity distribution from the surface of the sea to $40 \mathrm{~m}$ under water. The tidal current data were collected from a real ocean environment in China. From Figure 1, it can be seen that the tidal current velocity varies greatly at some adjacent depths, which is mainly caused by the turbulent flow affection. Consequently, the prediction model, by applying the multilayer tidal current velocity difference as input, enables an increase in prediction accuracy in contrast to only using a single tidal current velocity as input, as shown in Figure 2.

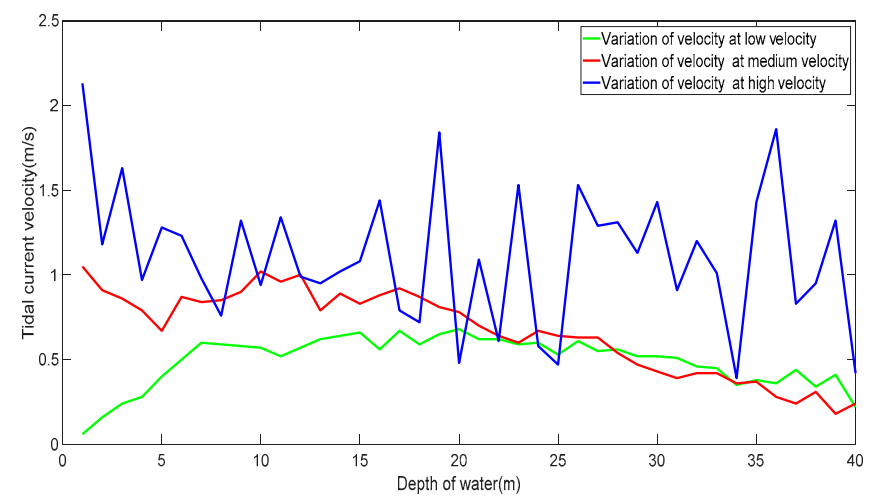

Figure 1. Distribution diagram of tidal current velocity distribution from the surface of the sea to $40 \mathrm{~m}$ under the sea surface. The green line presents the tidal current at low velocity condition; the red line presents the tidal current at medium velocity condition; the blue line presents the tidal current at high velocity condition.

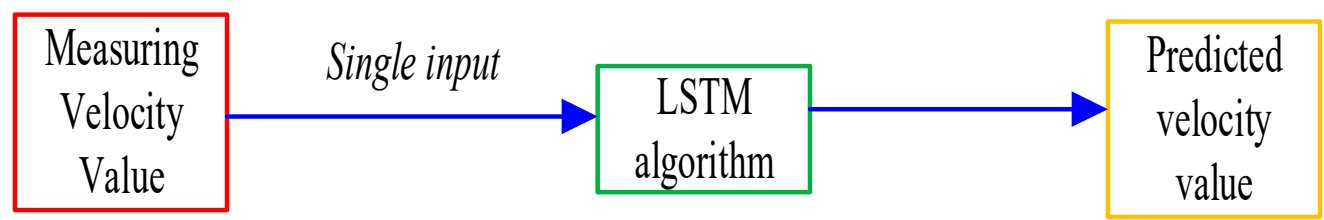

Figure 2. Diagram of a traditional single input tidal current prediction method.

As above-mentioned, a novel tidal current prediction considering the effect of the multi-layer current velocity method was proposed. Figure 3 shows a diagram of the novel tidal current prediction considering the effect of the multi-layer current velocity method. The LSTM algorithm has an advantage of capturing long-term dependencies within a sequence. This feature of LSTM makes it suitable to be applied in tidal current prediction because the main component of tidal currents is a kind of sequential periodic wave. The tidal current velocity in the $m$-th layer ( $m$ means the depth of the predicted tidal current velocity) was selected as one of the LSTM prediction model inputs, which obtains the periodic components of tidal current velocity. Periodic components are the main components in tidal currents, which are generated by the astronomic factor. In terms of the random factor, the difference of tidal current velocity between the $m$-th layer and $n$-th layer (n means the depth adjacent to $m$-th layer) is used as the other prediction model input, which obtains the random components caused by the random factor. This is able to decrease the effect of the multilayer tidal current velocity difference 
caused by the effect of turbulent flow (random components of tidal current velocity). In contrast to the traditional single input tidal current prediction method, a novel tidal current prediction includes both the astronomic factor and random factor by considering the effect of multi-layer current velocity.

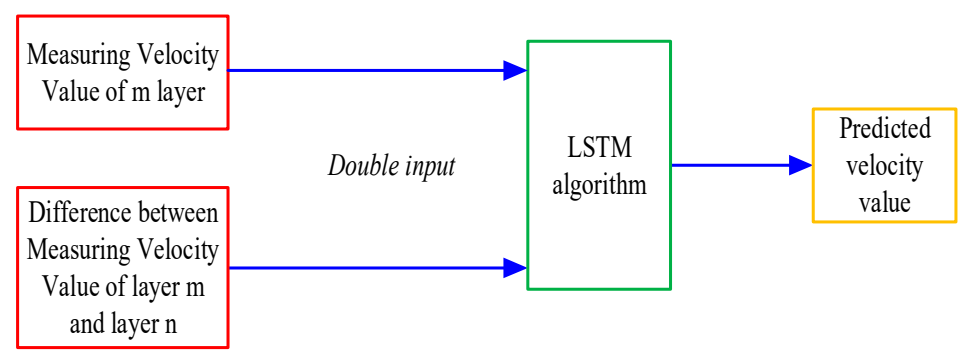

Figure 3. Diagram of a novel tidal current prediction considering the effect of the multi-layer current velocity method.

\subsection{Harmonic Analysis Method}

Essentially, the main principle of the HA method is the data analysis of tides generated by changes in the gravitational force. The magnitude of gravity is mainly dependent on the relative positions of the Earth, the Moon, and the Sun. Hence, the effects of different positions are combined with a set of particular components. Selecting a set of time series observations: $y(t), t=t_{1}, t_{2} \ldots, t_{M}$, the time interval is $\Delta t$. Then, the tidal model with $N$ constituents is described as follows:

$$
x(t)=b_{0}+b_{1} t+\sum_{k=1, \ldots, N} a_{k} e^{i \sigma_{k} t}+a_{-k} e^{-i \sigma_{k} t}
$$

where the $a_{k}$ and $a_{-k}$ are the variation of the constituents; $\sigma_{k}$ is the frequency of each constituent; $b_{0}$ is an offset; and $b_{1}$ indicates the trend.

In this paper, the least squares technique was adopted in the HA based analysis due to it being more flexible and accurate.

The least squares equation is given as follows:

$$
E=\sum_{M}\left|x\left(t_{m}\right)-y\left(t_{m}\right)\right|^{2}=|| T a-y||
$$

where $y=\left[y\left(t_{1}\right), y\left(t_{2}\right), \ldots, y\left(t_{M}\right)\right]^{\prime} ; a=\left[b_{0}, b_{1}, a_{1}, a_{-1}, a_{2}, a_{-2}, \ldots, a_{-N}\right]^{\prime} ;$ and $T$ is a $(M \times 2 N+2)$ matrix of linear and sinusoidal basis functions evaluated at observation times.

The standard parameters of the particular constituent $k$ are then calculated as follows:

$$
\begin{gathered}
L_{k}=\left|a_{k}\right|+\left|a_{-k}\right| \\
l_{k}=\left|a_{k}\right|-\left|a_{-k}\right| \\
\theta_{k}=\frac{\operatorname{ang}\left(a_{k}\right)+\operatorname{ang}\left(a_{-k}\right)}{2} \\
g_{k}=v_{k}-\operatorname{ang}\left(a_{k}\right)+\theta_{k}
\end{gathered}
$$

where $L_{k}$ and $l_{k}$ are the semi-major axis and semi-minor axis of the ellipse respectively; $\theta_{k}$ is the inclination angle of the semi-major axis; and $g_{k}$ is the Greenwich phase.

The constituent selection method was proposed by Foreman and Rayleigh, which is used to achieve automatic constituent selection. Generally, the constituent selection is an iterative process. The classical approach of the constituent selection criterion is the Rayleigh criterion proposed by Foreman in 1977. The Rayleigh criterion illustrates two constituents (frequencies $\omega_{q 1}$ and $\omega_{q 2}$ ) that are decomposed by a record with uniformly distributed times. 
In terms of raw input with length of record $(L O R) t_{n_{t}}-t_{1}$; the frequency resolution is $\Delta \omega=1 / L O R_{e}$.

$$
R^{R}\left(q_{1}, q_{2}\right)=\left(\frac{L O R_{e}}{\frac{1}{\left|\omega_{q 2}-\omega_{q 1}\right|}}\right) / R_{\text {min }} \geq 1
$$

where $R_{\min }$ is a minimum threshold; when $R_{\min }=1$, the criterion is equivalent to obtain a sufficiently long record length, by comparing all constituent pairs and omitting constituents, the suitable constituents of the raw input can be obtained.

A commercial harmonic analysis toolbox UTide [31], proposed by Codiga in 2011, enables the analysis of harmonic currents. UTide integrates various harmonic analysis methods, which is specifically designed to treat multi-year records with irregular temporal sampling.

\subsection{Machine Learning Based Prediction Approaches}

\subsubsection{Long-Short Term Memory}

The LSTM algorithm is an improved RNN algorithm. With the unique memory and forgotten pattern, the LSTM algorithm can understand the timing characteristics and the time-dependent relation accurately [32,33]. The LSTM algorithm is able to preferably solve the problems of gradient descent and gradient explosion that are inherent disadvantages of traditional circulating neural networks [34]. As the number of network layers increases, the perception of subsequent nodes to the previous nodes is weakened, which makes the model lose the previous information phenomenon over time. The LSTM algorithm has therefore been applied in many application fields including natural language translation, image processing, and voice recognition. Compared with traditional RNN algorithm, LSTM algorithm has three types of gates (input gate, forget gate, and output gate) and a memory cell with the LSTM neuron.

The LSTM neuron can achieve the function of information discarding and retaining via transferring information with gate structure.

According to Figure 4, first, the forget gate chooses information for discarding. The output value $h_{t-1}$ from the last time output and the new input information $X_{t}$ are used as inputs. Through the activation function sigmoid, they are mapped between 0 and 1 . The function of the forget gate is given as follows:

$$
f_{t}=\operatorname{sigmoid}\left(W_{h f} h_{t-1}+W_{x f} X_{t}\right)
$$

where $W_{h f}$ and $W_{x f}$ are the weights, and sigmoid is an activation function:

$$
\text { sigmoid }=\frac{1}{1+\mathrm{e}^{-\mathrm{x}}}
$$

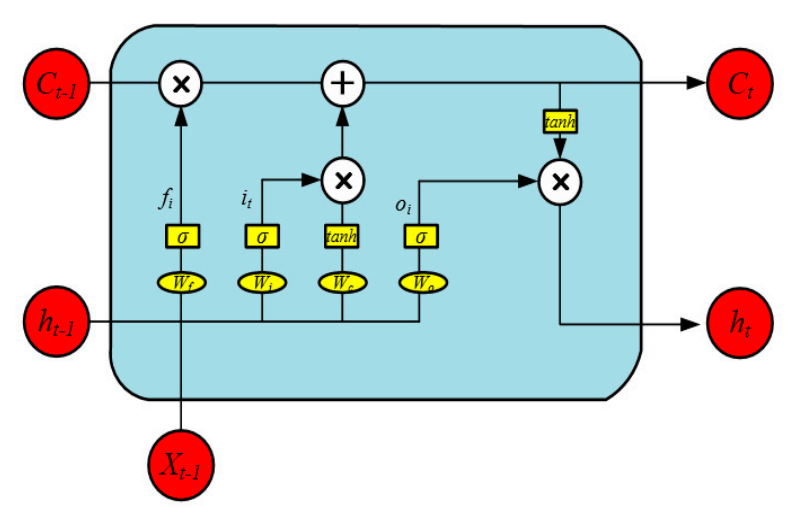

Figure 4. Structure of the long short-term memory (LSTM) algorithm. 
Second, the input gate chooses information for retaining. The output value $h_{t-1}$ from the last time output and the new input information $X_{t}$ are selected as inputs. Through the activation function sigmoid, they are mapped between 0 and 1 . The function of the input gate is given as follows:

$$
i_{t}=\operatorname{sigmoid}\left(W_{h f} h_{t-1}+W_{x f} X_{t}\right)
$$

Aside from the above-mentioned functions, there is a new vector $C_{t}^{\prime}$, which represents all information.

$$
C_{t}^{\prime}=\tanh \left(W_{h c} h_{t-1}+W_{x c} X_{t}\right)
$$

where $W_{h c}$ and $W_{x c}$ are the weights, and $\tanh (x)=2 \operatorname{sigmoid}(2 x)-1$.

With information adding and forgetting, the state of the cell can be updated from $C_{t-1}$ to $C_{t}$.

$$
C_{t}=f_{t} C_{t-1}+i_{t} C_{t}^{\prime}
$$

New cell state $C_{t}$ is composed of state $C_{t-1}$ and the memorized information.

Finally, there are two steps to get output value $h_{t}$, described as follows:

$$
\begin{gathered}
O_{t}=\operatorname{sigmoid}\left(W_{h o} h_{t-1}+W_{x c} X_{t}+b_{o}\right) \\
h_{t}=O_{t} \tanh \left(C_{t}\right)
\end{gathered}
$$

where $W_{h o}$ and $W_{x c}$ are the weights, and $b_{o}$ is the offset.

From the information processing procedure, it can be seen that the output information is not only related to the input information, but also to the memory information of the cell state and the output information from the last time. It is a cumulative process among cell states, which solves the problems of vanishing gradient and exploding gradient.

The prediction model of LSTM with time series is shown in Figure 5, where it consists of the input layer, LSTM layer, two hidden layers, and output layer.

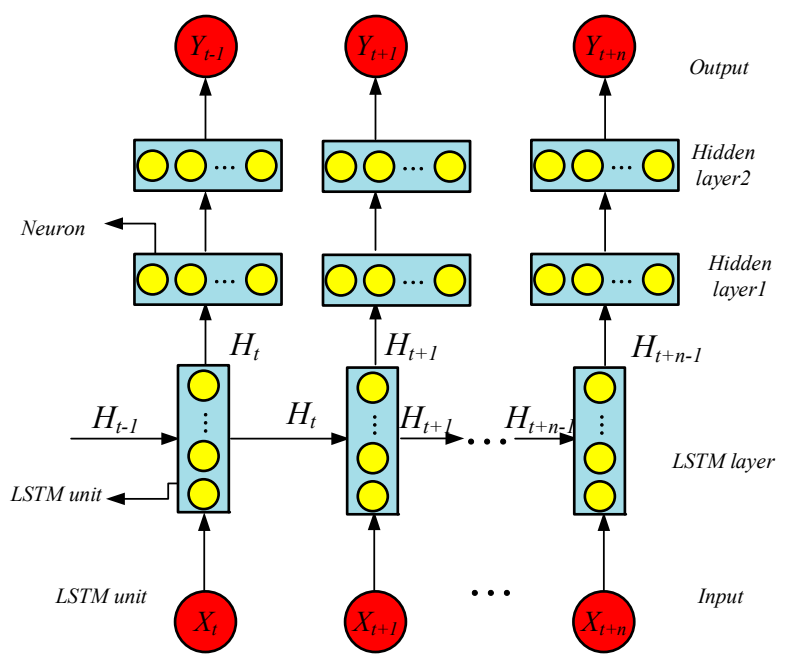

Figure 5. Prediction model of the LSTM.

The input layer is used to get the original information of the time series; the LSTM layer, composed of 48 LSTM units, is used to learn the time series relationship and extract features from inputs; two hidden layers, composed of 24 neurons and 12 neurons, respectively, are used to reduce dimensionality and map the feature learned from the LSTM layer; and finally, the results are obtained from the output layer. 
According to the model structure above-mentioned, the prediction results of non-stationary sequences can be expressed as follows:

$$
\mathrm{y}_{\mathrm{t}}^{\prime}=w_{3}\left(w_{2}\left(\operatorname{relu}\left(w_{1} H_{t}+b_{1}\right)\right)+b_{2}\right)+b_{3}
$$

where $w_{1}, w_{2}, w_{3}$, are the weights of the LSTM layer, hidden layer and output layer; and $H_{t}$ $\left(h_{t 1}, h_{t 2}, \ldots, h_{t s}\right)$ is the output state of LSTM cells at time $t$. Relu is an activation function: $f(x)=\max (0, x)$.

\subsubsection{Back-Propagation Artificial Neural Network}

Among the numerous ANN methods, a single hidden layer feed forward neural network (SLFN) using the gradient-based back-propagation (BP) training algorithm, is one of the most classical ANN methods. It is usually selected as a standard of comparison for estimating the performance of other ANN methods.

Figure 6 shows a diagram of a feed forward neural network with a single hidden layer. The network consists of an input layer, a hidden layer, and an output layer of neurons. For this example, the input layer has $n$ neurons; the hidden layer has $L$ neurons, and the output layer has $m$ neurons. Finally, $x_{1}$, $x_{2}, \cdots, x_{n}$ are the inputs to the network and $y_{1}, y_{2}, \cdots, y_{m}$ are the outputs from the network.

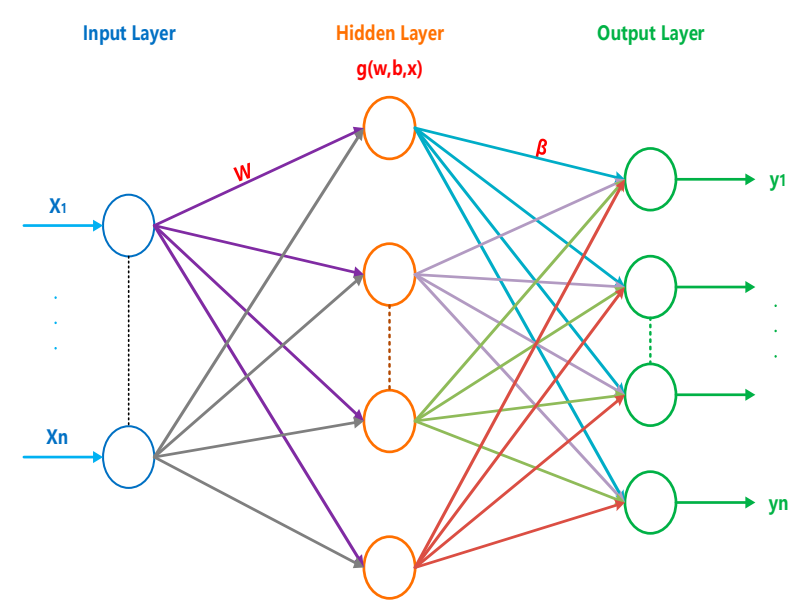

Figure 6. Schematic diagram of the single hidden layer feed forward neural network (SLFN) structure.

The parameters of the neural network structure are determined by bias values, activation constant, learning rate, and the number of hidden neurons. The optimal values of the neural network parameters are obtained by trial and error. In terms of the neural network structure optimization, it is essentially an optimization of the weight values. The steepest descent method is generally adopted to achieve weight value optimization.

\subsubsection{Elman Regression Network}

Unlike BPANN, the Elman neural network is a typical dynamic neural network, which is based on the structure of BPANN. By storing the internal state, it has the function of mapping dynamic characteristics. Hence, an Elman neural network has the ability to adapt to time-varying characteristics.

Compared with traditional BPANN, the Elman neural network has a receiving layer that is mainly used for feedback connection within or between layers. The Elman neural network is therefore able to achieve the time delay between input and output. With the description of dynamic equations, the Elman neural network has a memory function. The structure of the Elman neural network is shown in Figure 7. In terms of feedforward connection, the connection weights can be modified due to the existence of the receiving layer, while the weights of recursive are fixed. 


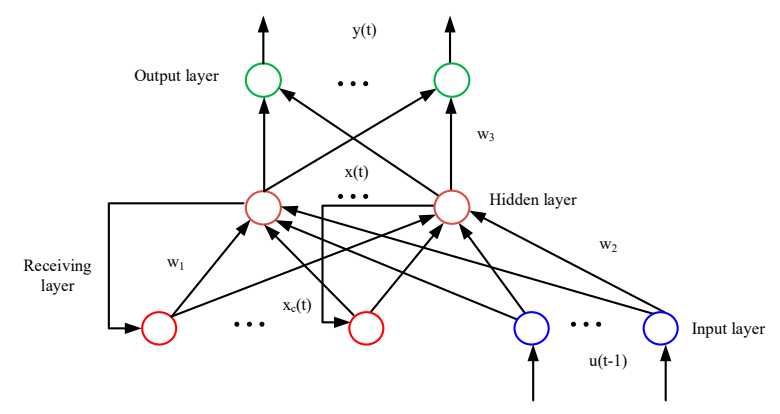

Figure 7. Schematic diagram of the Elman structure.

\section{Results}

The tidal current data were collected in Zhoushan, China. There was a consecutive observation of the velocity and the direction of the tidal current by using an acoustic doppler current profiler (ADCP) $[30,35]$. The ADCP equipment height was $1 \mathrm{~m}$, and the distance between the top and the center of the first measurement unit was $1.5 \mathrm{~m}$. The water depth can be roughly obtained through water pressure measurement, and atmospheric pressure was compensated during the measurement process. The sampling parameters of ADCP are listed as Table 1.

Table 1. The sampling parameters of acoustic doppler current profiler (ADCP).

\begin{tabular}{ll}
\hline Measurement Time & UTC+8 2018/2/13 12:00-2018/4/11 12:40 \\
\hline Measurement location & UTC+8 2018/2/13 12:00-2018/4/11 12:40 (Five minutes interval) \\
\hline ADCP model & Nortek AWAC 600 kHz \\
\hline Sampling frequency & $1 \mathrm{~Hz}$ (Continuous measurement for $30 \mathrm{~s}$ every $5 \mathrm{~min}$ ) \\
\hline Ping & Continuous measurement for 30 s every 5 min \\
\hline Pulse frequency & $600 \mathrm{kHz}$ \\
\hline Unit height (bin) & $1 \mathrm{~m}$ \\
\hline Number of units (bin) & 40 \\
\hline
\end{tabular}

The measurement time lasted from February 13 to 20, and the pressure value indicated that the water depth was about $40 \mathrm{~m}$. There are three steps in the post processing acquisitions: (1) remove data during deployment and recovery; (2) remove the echo intensity values below the threshold value; and (3) remove outliers such as sudden changes in tidal current data.

In order to validate and compare the performance of the above-mentioned methods, the mean absolute error (MAE) and the root-mean-squared error (RMSE) were used to evaluate the prediction accuracy, which is defined as follows:

$$
\begin{gathered}
M A E=\frac{1}{n} \sum_{i=1}^{n}\left|\overline{\varphi_{i}}-\varphi_{i}\right|^{2} \\
R M S E=\sqrt{\frac{1}{n} \sum_{i=1}^{n}\left(\overline{\varphi_{i}}-\varphi_{i}\right)^{2}}
\end{gathered}
$$

where $\overline{\varphi_{i}}$ is the forecast data, while $\varphi_{i}$ is the measurement data.

\subsection{Case Study A (20 m under Sea Surface)}

\subsubsection{Single Input Tidal Current Prediction Method}

In case study A, a short-term (with one-hour prediction interval) tidal current prediction was applied. Four types of algorithms were adopted to compare the performance in tidal current prediction. 
The commercial tidal current prediction software (UTide) adopts the harmonic analysis method to obtain the periodic components of tidal current velocity. The tidal data collected from $20 \mathrm{~m}$ under the sea surface were selected as input for UTide data analysis. The other three machine learning based algorithms (LSTM, BPANN, and ELMAN) were applied in the single input prediction method. Figures 8 and 9 show the comparison results of the single input prediction method by using three machine learning algorithms and UTide (20 $\mathrm{m}$ under sea surface). Through these comparison results, it can be seen that the predicted outputs of UTide were smooth periodic signals that lacked random components, which affects the prediction accuracy, while the LSTM algorithm had much better performance than the other two machine learning algorithms.

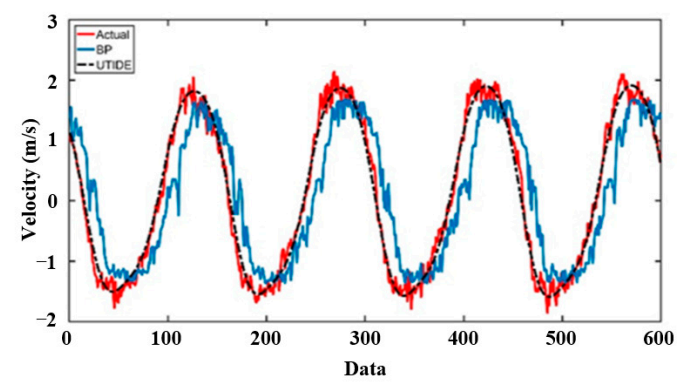

(a)

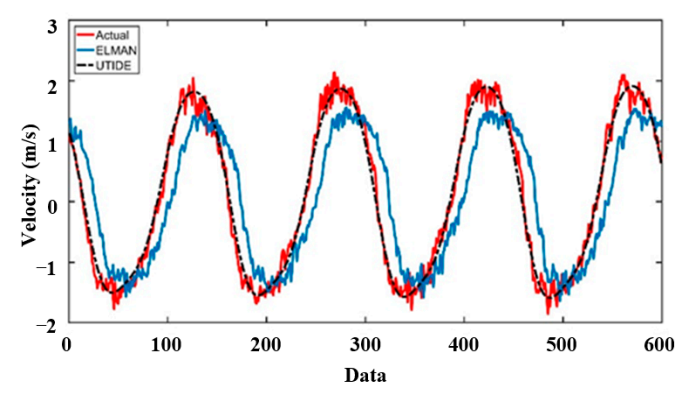

(b)

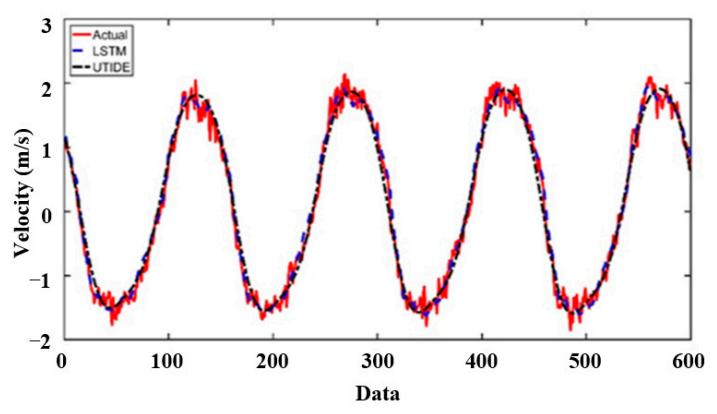

(c)

Figure 8. Comparison results of the single input prediction method by using three machine learning algorithms and UTide (20 m under sea surface). (a) Comparison of the back-propagation (BP) method, UTide method, and actual value, (b) Comparison of the ELMAN method, UTide method, and actual value, (c) Comparison of the LSTM method, UTide method, and actual value.

In order to further compare the performance of each method, residual signals of the single input prediction method by using three machine learning algorithms and the UTide analysis results are given in Figure 10. The residual signal is the difference between the prediction result and actual measurement data. It can be seen that the UTide and LSTM algorithms had higher accuracy than the other two 
algorithms. The prediction errors of the UTide and LSTM algorithms were under $\pm 0.5 \mathrm{~m} / \mathrm{s}$. In terms of RMSE and MAE values, the values of UTide were 0.214 and 0.165 , respectively, while the values of the LSTM algorithm were 0.142 and 0.113 , respectively. This means that the LSTM algorithm had the highest accuracy among the four types of algorithms.

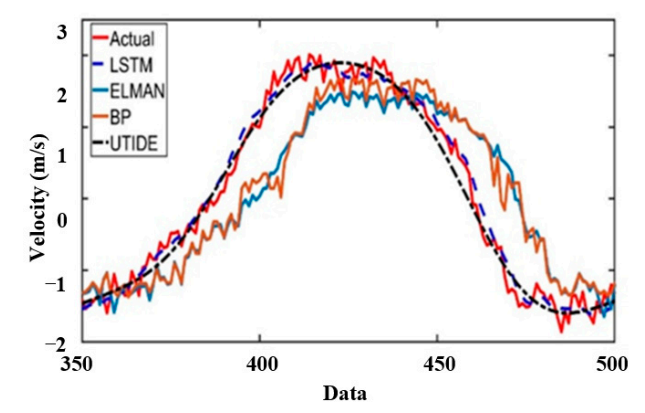

Figure 9. Enlarged figure of the comparison results in Figure 8.

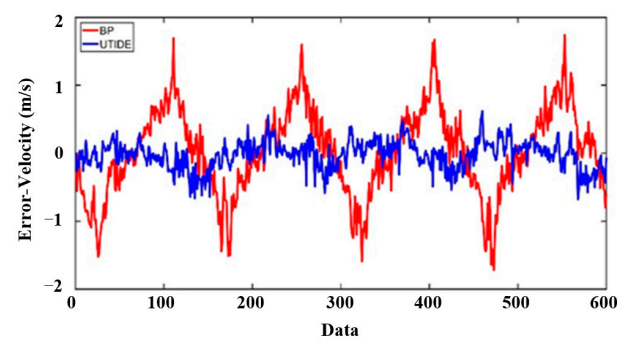

(a)

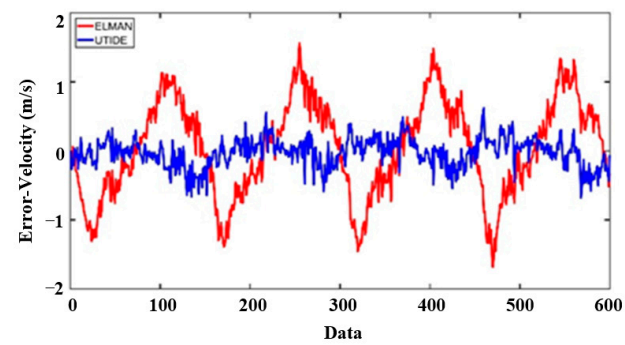

(b)

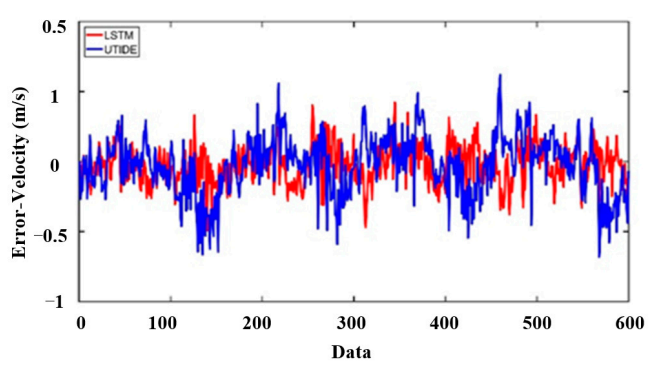

(c)

Figure 10. Residual signals of the single input prediction method by using three machine learning algorithms and UTide (20 m under sea surface). (a) Residual signals by using BP and UTide, (b) Residual signals by using ELMAN and UTide, (c) Residual signals by using LSTM and UTide.

4.1.2. Tidal Current Velocity Prediction Considering Effect of Multi-Layer Current Velocity Method

Apart from the traditional single prediction input method, the proposed tidal current velocity prediction considering the effect of the multi-layer current velocity method was also performed to 
validate the effectiveness. For input variables, the tidal current velocity at $20 \mathrm{~m}$ depth of the sea and the tidal current velocity difference (between $21 \mathrm{~m}$ depth of the sea and $20 \mathrm{~m}$ depth of the sea) were used as inputs of the proposed prediction method. Figures 11 and 12 illustrate the comparison results of the proposed method by using three machine learning algorithms and UTide $(20 \mathrm{~m}$ under sea surface). Through these comparison results, it can be seen that the LSTM algorithm has much better performance than the other two machine learning algorithms.

In order to further compare the performance of each method, residual signals of the proposed method by using three machine learning algorithms and UTide are given in Figure 13. It can be seen that the UTide and LSTM algorithms had higher accuracy than the other two algorithms. The prediction errors of the UTide and LSTM algorithms were under $\pm 0.5 \mathrm{~m} / \mathrm{s}$. In terms of RMSE and MAE values, the values of the LSTM algorithm were 0.125 and 0.099 , respectively. This means that the LSTM algorithm had the highest accuracy among the four types of algorithms. Furthermore, the proposed tidal current velocity prediction considering the effect of the multi-layer current velocity method had higher prediction accuracy than the traditional single input prediction method.

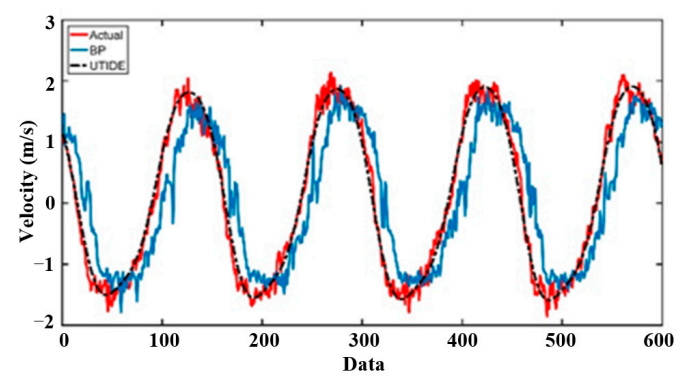

(a)

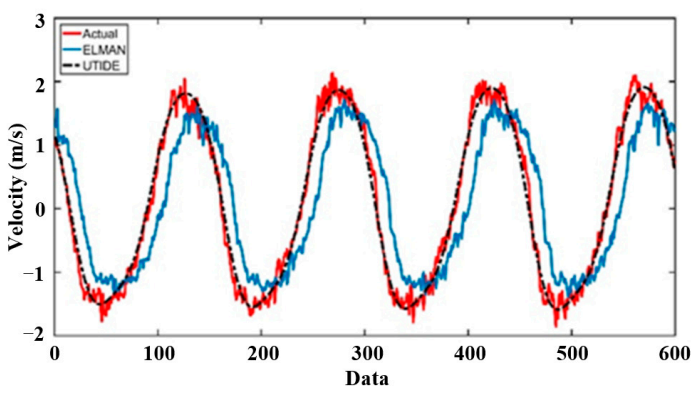

(b)

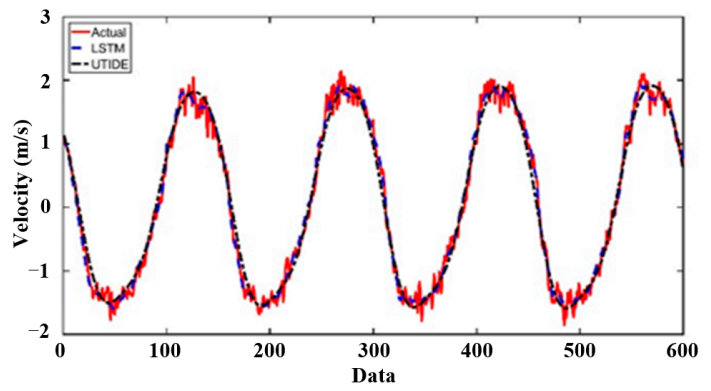

(c)

Figure 11. Comparison results of the proposed method by using three machine learning algorithms and UTide (20 $\mathrm{m}$ under sea surface). (a) Comparison of the BP method, UTide method, and actual value, (b) Comparison of the ELMAN method, UTide method, and actual value, (c) Comparison of LSTM method, UTide method and actual value. 


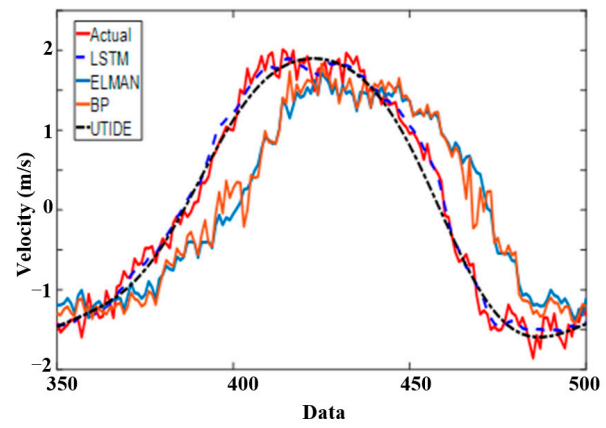

Figure 12. Enlarged figure of the comparison results in Figure 11.

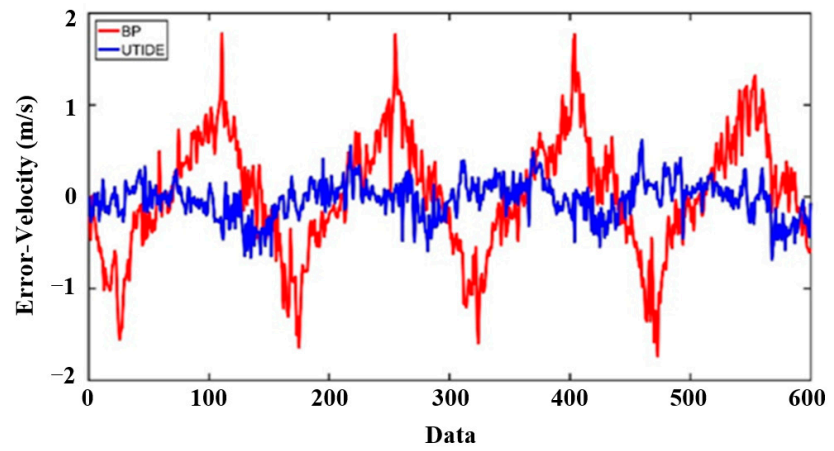

(a)

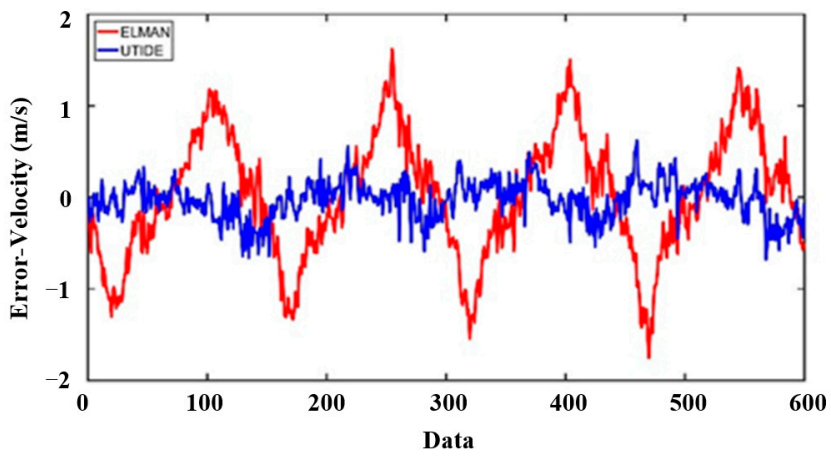

(b)

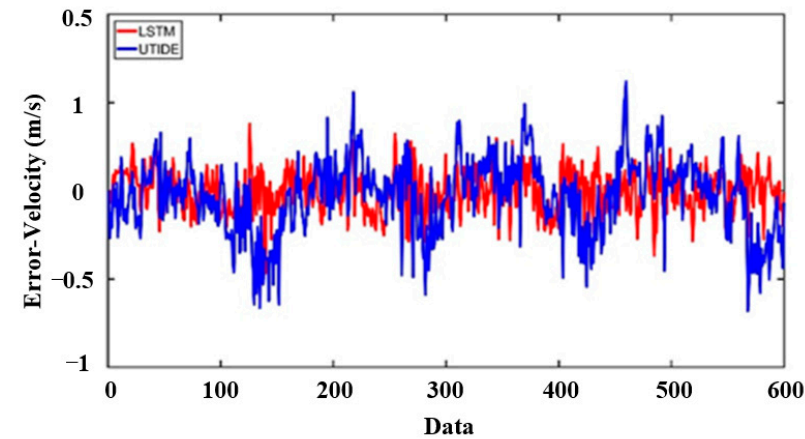

(c)

Figure 13. Residual signals of the proposed method by using three machine learning algorithms and UTide (20 m under sea surface). (a) Residual signals by using BP and UTide, (b) Residual signals by using ELMAN and UTide, (c) Residual signals by using LSTM and UTide. 


\subsection{Case Study B (30 m under Sea Surface)}

\subsubsection{Single Input Tidal Current Prediction Method}

The proposed method was also applied at different depths of the sea (30 $\mathrm{m}$ under the sea). Four types of algorithms were adopted to compare the performance in tidal current prediction. Figures 14 and 15 show the comparison results of the single input prediction method with three machine learning algorithms and UTide (30 m under sea surface). Through these comparison results, it can be seen that the LSTM algorithm had much better performance than the other two machine learning algorithms.

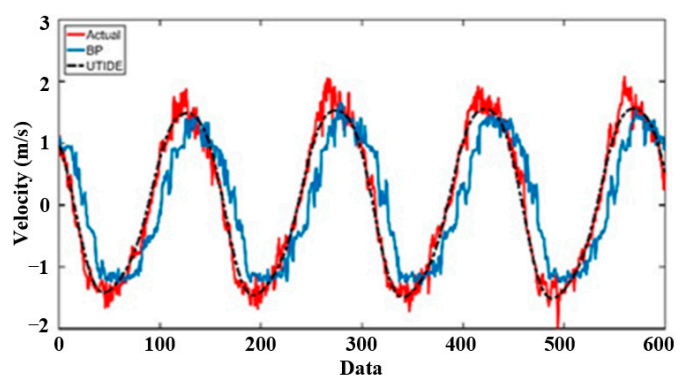

(a)

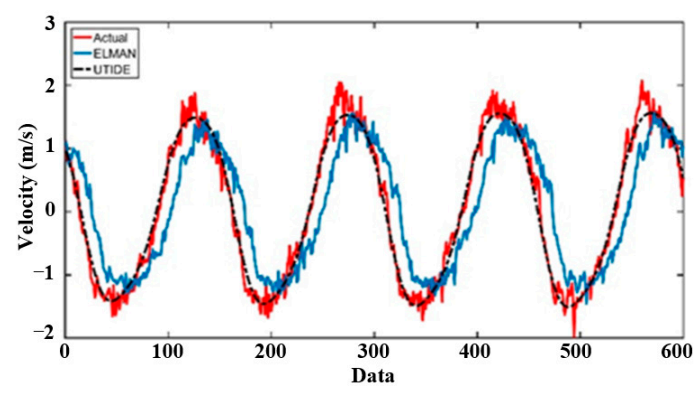

(b)

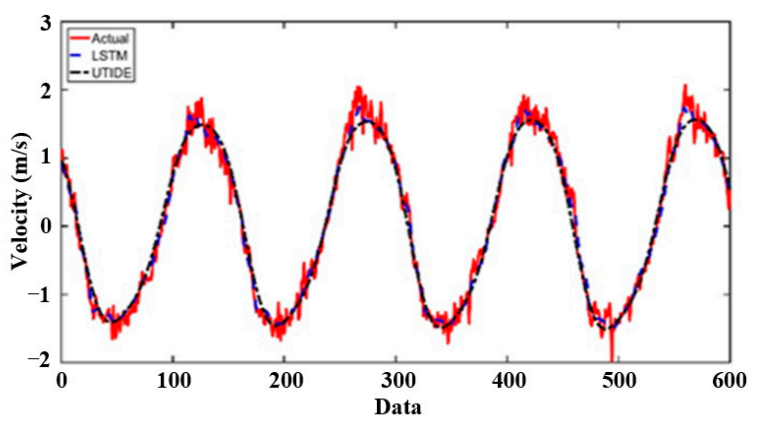

(c)

Figure 14. Comparison results of the single input prediction method by using three machine learning algorithms and UTide (30 m under sea surface). (a) Comparison of the BP method, UTide method, and actual value, (b) Comparison of the ELMAN method, UTide method, and actual value, (c) Comparison of the LSTM method, UTide method, and actual value.

In order to further compare the performance of each method, residual signals of the single input prediction method with three machine learning algorithms and UTide analysis results are given in Figure 16. It can be seen that the UTide and LSTM algorithms had higher accuracy than the other two algorithms. The prediction errors of the UTide and LSTM algorithms were under $\pm 0.5 \mathrm{~m} / \mathrm{s}$. In terms of RMSE and MAE values, values of UTide were 0.177 and 0.138 , respectively, while values of the LSTM 
algorithm were 0.145 and 0.115 , respectively. This means that the LSTM algorithm had the highest accuracy among the four types of algorithms.

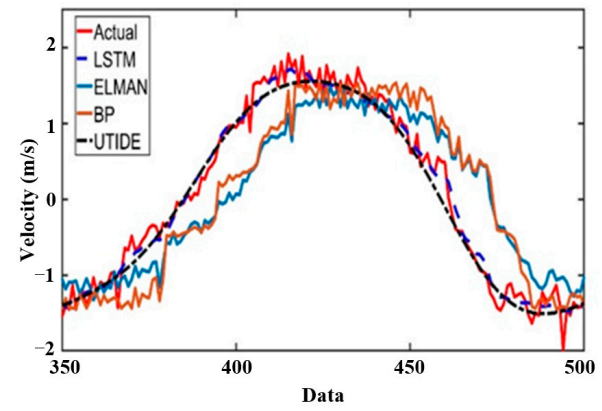

Figure 15. Enlarged figure of the comparison results in Figure 14.

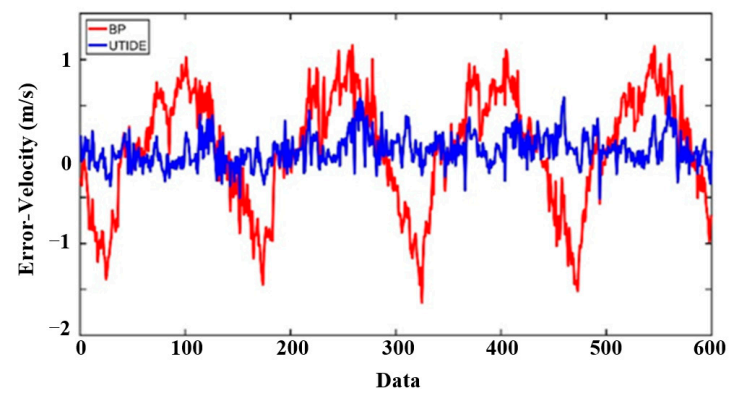

(a)

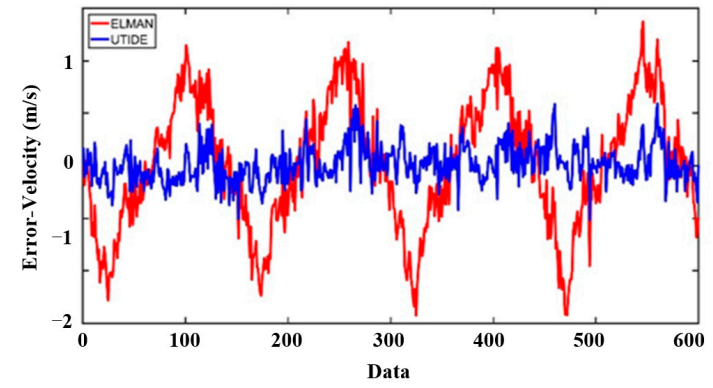

(b)

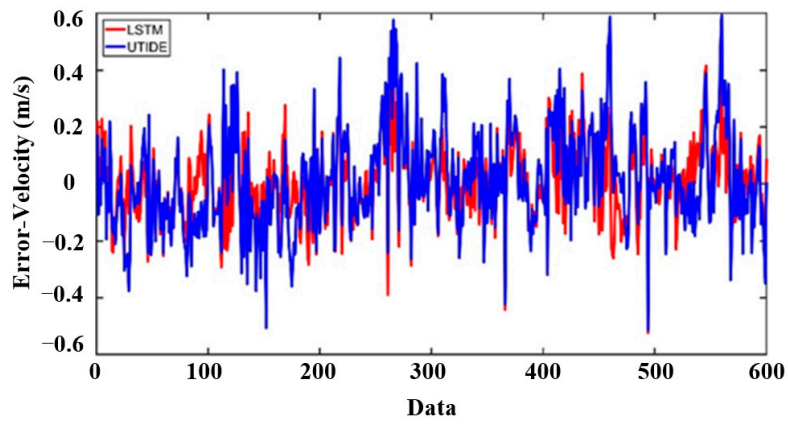

(c)

Figure 16. Residual signals of single input prediction method by using three machine learning algorithms and UTide (30 m under sea surface). (a) Residual signals by using BP and UTide, (b) Residual signals by using ELMAN and UTide, (c) Residual signals by using LSTM and UTide. 
4.2.2. Tidal Current Velocity Prediction Considering Effect of the Multi-Layer Current Velocity Method

Apart from the traditional single prediction input method, the proposed tidal current velocity prediction considering the effect of the multi-layer current velocity method was also performed to validate the effectiveness. For input variables, the tidal current velocity at $30 \mathrm{~m}$ depth of the sea and the tidal current velocity difference (between $31 \mathrm{~m}$ depth of the sea and $30 \mathrm{~m}$ depth of the sea) were used as inputs of the proposed prediction method. Figures 17 and 18 illustrate the comparison results of the proposed method with three machine learning algorithms and UTide (30 m under sea surface). Through these comparison results, it can be seen that the LSTM algorithm had much better performance than the other two machine learning algorithms.

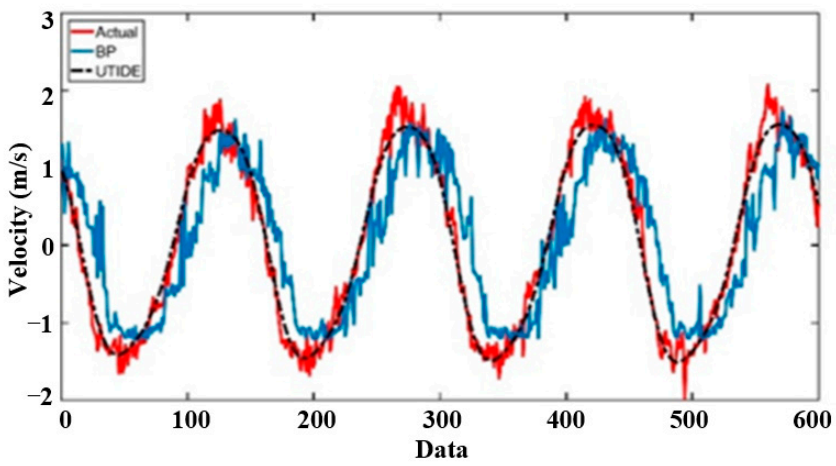

(a)

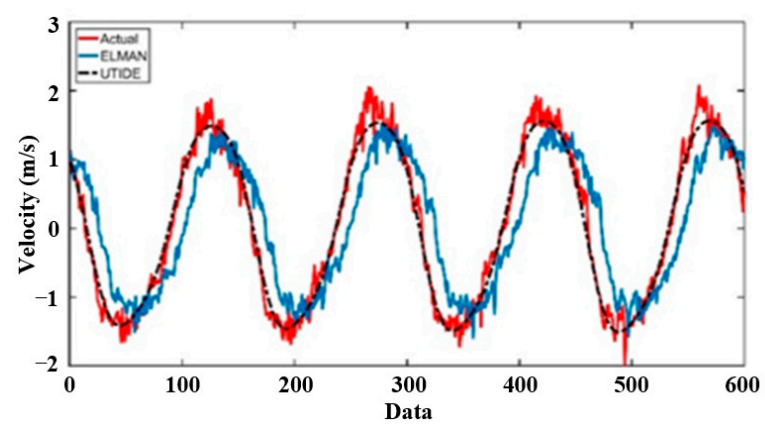

(b)

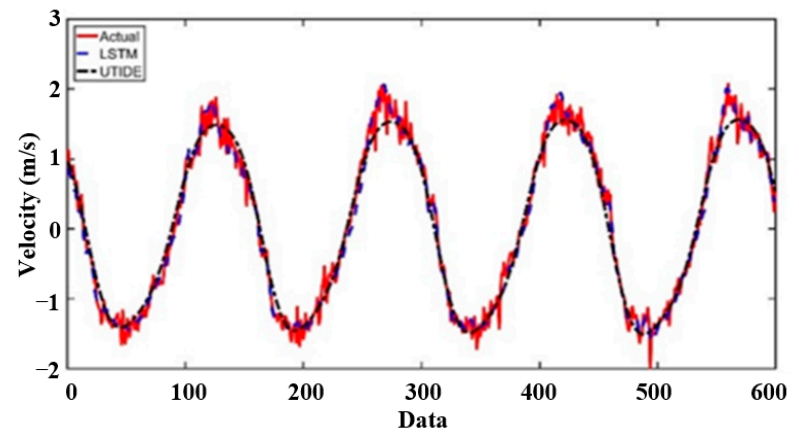

(c)

Figure 17. Comparison results of the proposed method by using three machine learning algorithms and UTide (30 m under sea surface). (a) Comparison of the BP method, UTide method, and actual value, (b) Comparison of the ELMAN method, UTide method, and actual value, (c) Comparison of the LSTM method, UTide method, and actual value. 


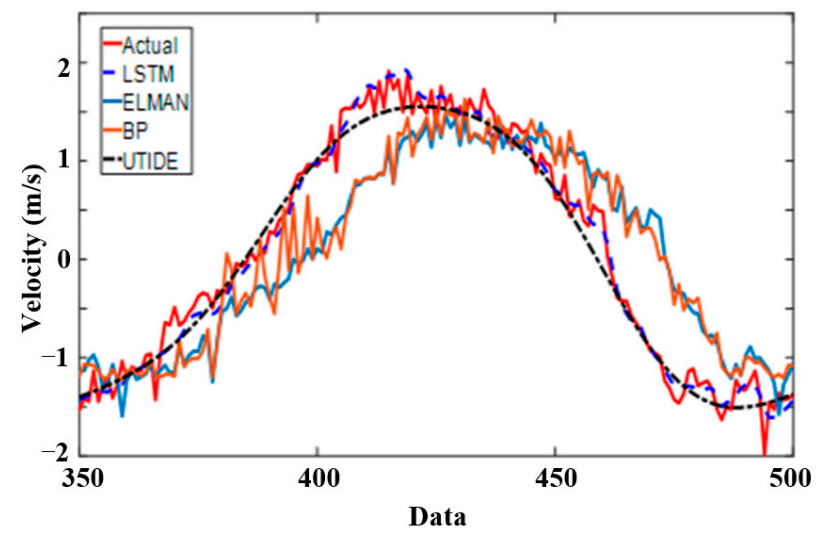

Figure 18. Enlarged figure of the comparison results in Figure 17.

In order to further compare the performance of each method, the residual signals of the proposed method by using three machine learning algorithms and UTide are given in Figure 19. It can be seen that the UTide and LSTM algorithms had higher accuracy than the other two algorithms. The prediction errors of the UTide and LSTM algorithms were under $\pm 0.5 \mathrm{~m} / \mathrm{s}$. In terms of RMSE and MAE values, values of the LSTM algorithm were 0.143 and 0.109 , respectively. This means that the LSTM algorithm had the highest accuracy among the four types of algorithms. Furthermore, the proposed tidal current velocity prediction, considering the effect of multi-layer current velocity method, had higher prediction accuracy than the traditional single input prediction method in this case study.

Detailed comparison results (RMSE and MAE evaluation) of the tidal current velocity prediction are illustrated in Tables 2 and 3. In these comparative investigations, the proposed prediction method, traditional single input prediction method, and UTide were performed by using two sets of tidal current data. The results showed that the proposed prediction method combined with the LSTM algorithm had the higher prediction accuracy than the other methods performed in this paper.

Table 2. Comparison RMSE and MAE of the tidal current velocity prediction $20 \mathrm{~m}$ under the sea surface.

\begin{tabular}{cccc}
\hline Method & & RMSE & MAE \\
\hline UTide & & 0.214 & 0.165 \\
\hline \multirow{3}{*}{ Single input prediction model } & LSTM & 0.142 & 0.113 \\
& BPANN & 0.637 & 0.512 \\
& ELMAN & 0.696 & 0.584 \\
\hline \multirow{3}{*}{ Multiple input prediction model } & LSTM & 0.125 & 0.099 \\
& BPANN & 0.635 & 0.510 \\
& ELMAN & 0.671 & 0549 \\
\hline
\end{tabular}

Table 3. Comparison RMSE and MAE of the tidal current velocity prediction $30 \mathrm{~m}$ under the sea surface.

\begin{tabular}{cccc}
\hline Method & & RMSE & MAE \\
\hline UTide & & 0.177 & 0.138 \\
\hline \multirow{3}{*}{ Single input prediction model } & LSTM & 0.145 & 0.115 \\
& BPANN & 0.615 & 0.505 \\
& ELMAN & 0.604 & 0.497 \\
\hline \multirow{3}{*}{ Multiple input prediction model } & LSTM & 0.143 & 0.109 \\
& BPANN & 0.6 & 0.486 \\
& ELMAN & 0.603 & 0.496 \\
\hline
\end{tabular}

From the above two case studies, it can be seen that residual signals of BPANN and ELMAN had a time shift in the time series. With the prediction interval decreasing, the prediction accuracy 
of BPANN and ELMAN had a significant increase. The RMSE values of BPANN and ELMAN could reach 0.23 and 0.26 when the prediction interval was $5 \mathrm{~min}$. This can further prove that the prediction performances of BPANN and ELMAN were poorer than the LSTM in tidal current prediction.

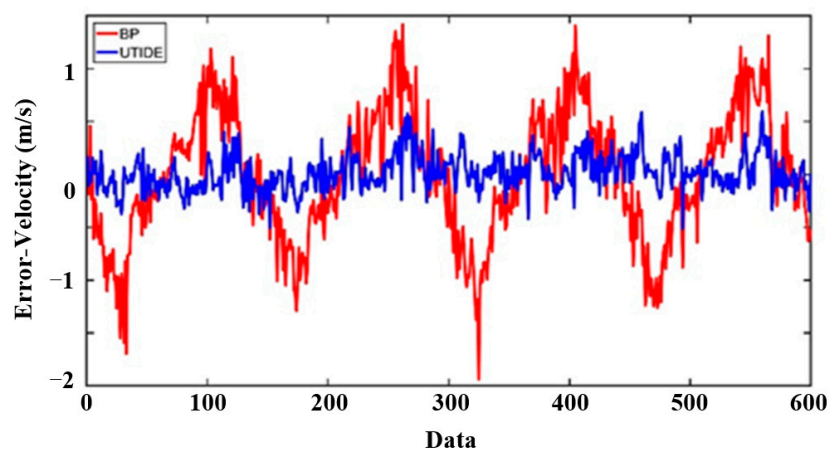

(a)

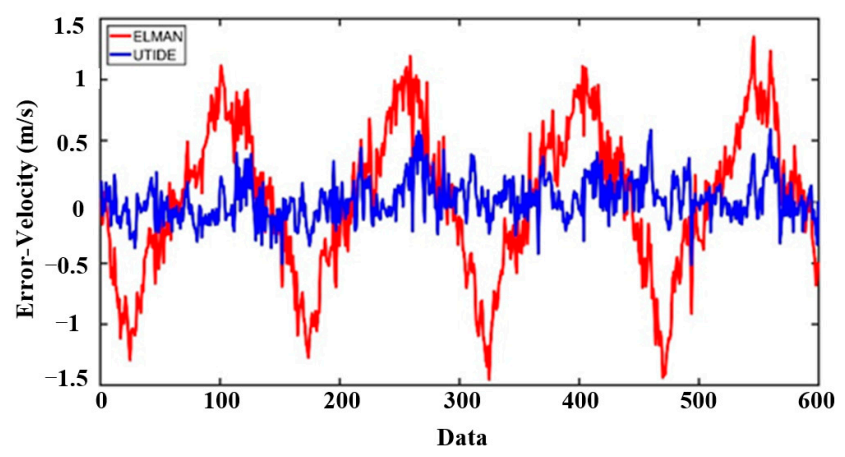

(b)

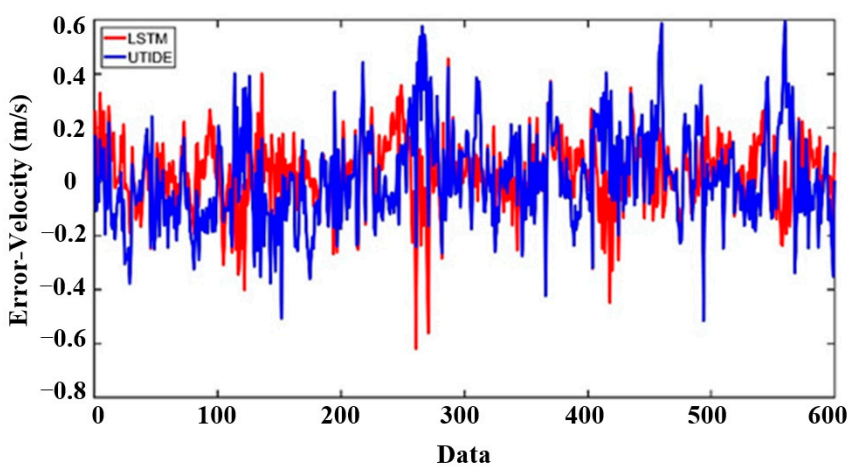

(c)

Figure 19. Residual signals of the proposed method by using three machine learning algorithms and UTide (30 m under sea surface). (a) Residual signals by using BP and UTide, (b) Residual signals by using ELMAN and UTide, (c) Residual signals by using LSTM and UTide.

\section{Conclusions}

The tidal current velocity data, collected from Zhejiang Province, were used to validate the effectiveness of the proposed method. Comparative investigations were carried out to demonstrate the accuracy of the tidal current velocity prediction method. Four types of algorithms were adopted to compare the performance in tidal current prediction. Through the data analysis results, two conclusions can be obtained: (1) The proposed tidal prediction method (shown in Figure 3) had higher prediction 
accuracy than the traditional single input tidal current prediction method (shown in Figure 2); and (2) the proposed method combined with the LSTM algorithm had the best performance among all the comparative investigation cases in this paper.

This article provides a new type of tidal current data processing and prediction method combined with tidal depth information. The proposed method effectively improved the accuracy of the tidal current velocity prediction and contributes to tidal current energy development, tidal current machine energy capture, and extreme sea state control and avoidance.

Future work will try to implement the online real time function of the proposed tidal current prediction method and develop a real time tidal current prediction system based on the proposed method.

Author Contributions: Conceptualization, P.Q.; Methodology, Y.S.; Investigation, H.W.; Resources, X.L.; Data Curation, H.Y.; Writing-Original Draft Preparation, B.F.; Writing-Review \& Editing, D.Z. All authors have read and agreed to the published version of the manuscript

Funding: This research was funded by National Natural Science Foundation of China (Grant Nos. 51879233 and 51705453), The International Collaboration and Exchange Program from the NSFC-RCUK/EPSRC (Grant No. 51761135011), and the Fundamental Research Funds for the Central Universities, and Research on Key Technologies of Marine Energy's Marine Equipment-Research on Key Technologies of Marine Energy's Marine Equipment for Smart Ocean from the Key Research and Development Program of Zhejiang Province (Grant No. 2021C03182).

Conflicts of Interest: The authors declare no conflict of interest.

\section{References}

1. Uihlein, A.; Magagna, D. Wave and tidal current energy-A review of the current state of research beyond technology. Renew. Sustain. Energy Rev. 2016, 58, 1070-1081. [CrossRef]

2. Ng, B. Tidal Current Predictions Using Rotary Empirical Orthogonal Functions. J. Atmos. Ocean. Technol. 1993, 10, 868-879. [CrossRef]

3. Vazquez, A.; Iglesias, G. LCOE (levelised cost of energy) mapping: A new geospatial tool for tidal stream energy. Energy 2015, 91, 192-201. [CrossRef]

4. Liu, M.; Li, W.; Wang, C.; Billinton, R.; Yu, J. Reliability Evaluation of a Tidal Power Generation System Considering Tidal Current Speeds. IEEE Trans. Power Syst. 2016, 31, 3179-3188. [CrossRef]

5. Morandeau, M.; Walker, R.T.; Argall, R.; Nicholls-Lee, R.F. Optimisation of marine energy installation operations. Int. J. Mar. Energy 2013, 3-4, 14-26. [CrossRef]

6. Qian, P.; Feng, B.; Liu, H.; Tian, X.; Si, Y.; Zhang, D. Review on configuration and control methods of tidal current turbines. Renew. Sustain. Energy Rev. 2019, 108, 125-139. [CrossRef]

7. Xu, Q.; Li, W.; Lin, Y.; Liu, H.; Gu, Y. Investigation of the performance of a stand-alone horizontal axis tidal current turbine based on in situ experiment. Ocean Eng. 2016, 113, 111-120. [CrossRef]

8. Wang, S.; Yuan, P.; Li, D.; Jiao, Y. An overview of ocean renewable energy in China. Renew. Sustain. Energy Rev. 2011, 15, 91-111. [CrossRef]

9. Zhang, D.; Liu, X.; Tan, M.; Qian, P.; Si, Y. Flow field impact assessment of a tidal farm in the Putuo-Hulu Channel. Ocean Eng. 2020, 208, 107359. [CrossRef]

10. Sun, C.; Lam, W.H.; Dai, M.; Hamill, G. Prediction of Seabed Scour Induced by Full-Scale Darrieus-Type Tidal Current Turbine. J. Mar. Sci. Eng. 2019, 7, 342. [CrossRef]

11. Darwin, G.H. Ellipsoidal Harmonic Analysis. In Philosophical Transactions of the Royal Society of London. Series A, Containing Papers of a Mathematical or Physical Character; Royal Society: London, UK, 1901; Volume 197, pp. 461-557.

12. Foreman, M.G.G.; Cherniawsky, J.Y.; Ballantyne, V.A. Versatile Harmonic Tidal Analysis: Improvements and Applications. J. Atmos. Ocean. Technol. 2009, 26, 806-817. [CrossRef]

13. Jin, G.; Pan, H.; Zhang, Q.; Lv, X.; Zhao, W.; Gao, Y. Determination of Harmonic Parameters with Temporal Variations: An Enhanced Harmonic Analysis Algorithm and Application to Internal Tidal Currents in the South China Sea. J. Atmos. Ocean. Technol. 2018, 35, 1375-1398. [CrossRef]

14. Jay, D.A.; Flinchem, E.P. A comparison of methods for analysis of tidal records containing multi-scale non-tidal background energy. Cont. Shelf Res. 1999, 19, 1695-1732. [CrossRef] 
15. Sarkar, D.; Osborne, M.A.; Adcock, T.A.A. Spatiotemporal Prediction of Tidal Currents Using Gaussian Processes. J. Geophys. Res. Oceans 2019, 124, 2697-2715. [CrossRef]

16. López, I.; Iglesias, G. Efficiency of OWC wave energy converters: A virtual laboratory. Appl. Ocean Res. 2014, 44, 63-70. [CrossRef]

17. Rodriguez-Delgado, C.; Bergillos, R.J.; Iglesias, G. An artificial neural network model of coastal erosion mitigation through wave farms. Environ. Model. Softw. 2019, 119, 390-399. [CrossRef]

18. Castro, A.; Carballo, R.; Iglesias, G.; Rabuñal, J.R. Performance of artificial neural networks in nearshore wave power prediction. Appl. Soft Comput. 2014, 23, 194-201. [CrossRef]

19. Lee, T.L.; Jeng, D.S. Application of artificial neural networks in tide-forecasting. Ocean Eng. 2002, 29, 1003-1022. [CrossRef]

20. Lee, T.-L. Back-propagation neural network for long-term tidal predictions. Ocean Eng. 2004, 31, $225-238$. [CrossRef]

21. Charhate, S.B.; Deo, M.C.; Kumar, V.S. Soft and hard computing approaches for real-time prediction of currents in a tide-dominated coastal area. Proc. Inst. Mech. Eng. Part M J. Eng. Marit. Environ. 2007, 221, 147-163. [CrossRef]

22. Qian, P.; Feng, B.; Zhang, D.; Tian, X.; Si, Y. IoT-based approach to condition monitoring of the wave power generation system. IET Renew. Power Gener. 2019, 13, 2207-2214. [CrossRef]

23. Sarkar, D.; Osborne, M.A.; Adcock, T.A.A. Prediction of tidal currents using Bayesian machine learning. Ocean Eng. 2018, 158, 221-231. [CrossRef]

24. Qian, P.; Ma, X.; Zhang, D.; Wang, J. Data-Driven Condition Monitoring Approaches to Improving Power Output of Wind Turbines. IEEE Trans. Ind. Electron. 2019, 66, 6012-6020. [CrossRef]

25. Chang, H.-K.; Lin, L.-C. Multi-point tidal prediction using artificial neural network with tide-generating forces. Coast. Eng. 2006, 53, 857-864. [CrossRef]

26. Graves, A. Long Short-Term Memory. In Supervised Sequence Labelling with Recurrent Neural Networks; Graves, A., Ed.; Springer: Berlin/Heidelberg, Germany, 2012; pp. 37-45.

27. Liu, F.; Cai, M.; Wang, L.; Lu, Y. An Ensemble Model Based on Adaptive Noise Reducer and Over-Fitting Prevention LSTM for Multivariate Time Series Forecasting. IEEE Access 2019, 7, 26102-26115. [CrossRef]

28. Sen, S.; Raghunathan, A. Approximate Computing for Long Short Term Memory (LSTM) Neural Networks. IEEE Trans. Comput. Aided Des. Integr. Circuits Syst. 2018, 37, 2266-2276. [CrossRef]

29. Balas Can, E.; Koç, L.; Balas, L. Predictions of Missing Wave Data by Recurrent Neuronets. J. Waterw. Port Coast. Ocean Eng. 2004, 130, 256-265. [CrossRef]

30. Cochin, V.; Mariette, V.; Broche, P.; Garello, R. Tidal Current Measurements Using VHF Radar and ADCP in the Normand Breton Gulf: Comparison of Observations and Numerical Model. IEEE J. Ocean. Eng. 2006, 31, 885-893. [CrossRef]

31. Codiga, D. Unified Tidal Analysis and Prediction Using the Utide Matlab Functions; Technical Report; Graduate School of Oceanography, University of Rhode Island: Narragansett, RI, USA, 2011.

32. Zhao, Z.; Chen, W.; Wu, X.; Chen, P.C.Y.; Liu, J. LSTM network: A deep learning approach for short-term traffic forecast. IET Intell. Transp. Syst. 2017, 11, 68-75. [CrossRef]

33. Ma, X.; Tao, Z.; Wang, Y.; Yu, H.; Wang, Y. Long short-term memory neural network for traffic speed prediction using remote microwave sensor data. Transp. Res. Part C Emerg. Technol. 2015, 54, 187-197. [CrossRef]

34. Lukoševičius, M.; Jaeger, H. Reservoir computing approaches to recurrent neural network training. Comput. Sci. Rev. 2009, 3, 127-149. [CrossRef]

35. Zhu, X.-H.; Ma, Y.-L.; Guo, X.; Fan, X.; Long, Y.; Yuan, Y.; Xuan, J.-L.; Huang, D. Tidal and residual currents in the Qiongzhou Strait estimated from shipboard ADCP data using a modified tidal harmonic analysis method. J. Geophys. Res. Ocean 2014, 119, 8039-8060. [CrossRef]

Publisher's Note: MDPI stays neutral with regard to jurisdictional claims in published maps and institutional affiliations. 